\title{
NOTE ON RINGS OF FINITE REPRESENTATION TYPE AND DECOMPOSITIONS OF MODULES
}

\author{
K. R. FULLER ${ }^{1}$ AND IDUN REITEN
}

ABSTRACT. Tachikawa has shown that if a ring $\mathbf{\Lambda}$ is of finite representation type, then each of its left and right modules has a decomposition that complements direct summands. We show that the converse is also true.

Anderson and Fuller [1] posed the problem of determining over which rings does every module have a decomposition $M=\bigoplus_{A} M_{a}$ that comple. ments direct summands in the sense that whenever $K$ is a direct summand of $M, M=K \oplus\left(\bigoplus_{B} M_{\beta}\right)$ for some $B \subset A$. In response, Tachikawa [6] has proved that the modules over a ring of finite representation type have such decompositions. We recall that an artin ring is of finite representation type if it has only a finite number of finitely generated indecomposable left modules. The purpose of this note is to use the results of [1]-[5] to show that the converse of Tachikawa's result is also true.

Auslander [3] says that a family of $R$-homomorphisms is noetherian if given a sequence

$$
M_{0} \stackrel{f_{0}}{\longrightarrow} M_{1} \stackrel{f_{1}}{\longrightarrow} M_{2} \rightarrow \cdots
$$

in the family, with $f_{i} \cdots f_{1} f_{0} \neq 0$ for all $i$, there is an integer $n$ such that $f_{k}$ is an isomorphism for all $k \geq n$, and that the family is conoetherian in case given any sequence

$$
\cdots \rightarrow M_{2} \stackrel{f_{1}}{\longrightarrow} M_{1} \stackrel{f_{0}}{\longrightarrow} M_{0}
$$

with $f_{0} f_{1} \cdots f_{i} \neq 0$ for all $i$, there is an integer $n$ such that $f_{k}$ is an isomorphism for all $k \geq n$. The result we seek follows from the following result of Auslander

Received by the editors March 12, 1974.

AMS (MOS) subject classifications (1970). Primary 16A46, 16A48, 16A64; Secondary 16A40.

Key words and phrases. Finite representation type, transpose, dual, decompositions of modules.

1Fuller's research was supported by NSF grant GP-18828. 
[3, Theorem 3.1]. If $\Lambda$ is a left artin ring over which the family of monomorphisms between finitely generated indecomposable left modules is noetherian and the family of epimorphisms between finitely generated indecomposable left modules is conoetherian then $\Lambda$ is a ring of finite representation type;

the following version of Harada and Sai's

[5, Lemma 9]. Let $\mathcal{F}$ be a family of finitely generated modules. If every $M=\bigoplus_{A} M_{a}$ with $M_{a} \in \mathcal{F}$ complements direct summands then the family of homomorphisms between the members of $\mathcal{F}$ is noetherian; and the

Proposition. Let $\Lambda$ be an artin ring. If the family of homomorphisms between finitely generated indecomposable right $\Lambda$-modules is noetherian then the family of epimorphisms between finitely generated indecomposable left $\Lambda$-modules is conoetherian.

Proof. The proof uses the Auslander-Bridger transpose [4] and some of its properties which can be found in [2] and [4] or derived by standard diagram chasing techniques. Let ()$^{*}=\operatorname{Hom}_{\Lambda}(-, \Lambda)$. I.et $M$ be a finitely generated left $\Lambda$-module that contains no nonzero projective direct summands, and let $P_{1} \stackrel{d}{\rightarrow} P_{0} \rightarrow M \rightarrow 0$ be a minimal projective resolution of $M$. Then the transpose of $M$ is a right $\Lambda$-module $\operatorname{Tr}(M)$ such that

$$
P_{0}^{*} \stackrel{d^{*}}{\longrightarrow} P_{1}^{*} \rightarrow \operatorname{Tr}(M) \rightarrow 0
$$

is exact. $\operatorname{Tr}(M)$ is also finitely generated with no nonzero projective direct summands, and $M$ is indecomposable iff $\operatorname{Tr}(M)$ is. If $N$ is another $\Lambda$-module with no projective direct summands and $f: M \rightarrow N$ is a homomorphism, there is a homomorphism $\hat{f}: \operatorname{Tr}(N) \rightarrow \operatorname{Tr}(M)$ satisfying:

(1) if $0 \neq f_{0} f_{1} \ldots f_{i}$ then $\hat{f}_{i} \ldots \hat{f}_{1} \hat{f}_{0} \neq 0$;

(2) $f$ is an isomorphism iff $\hat{f}$ is a.r isomorphism.

Thus a nonterminating sequence of proper epimorphisms between finitely generated indecomposable (necessarily nonprojective) left $\Lambda$-modules

$$
\cdots \rightarrow M_{2} \stackrel{f_{1}}{\longrightarrow} M_{1} \stackrel{f_{0}}{\longrightarrow} M_{0}
$$

yields a sequence

$$
\operatorname{Tr}\left(M_{0}\right) \stackrel{\hat{f}_{0}}{\longrightarrow} \operatorname{Tr}\left(M_{1}\right) \stackrel{\hat{f}_{1}}{\longrightarrow} \operatorname{Tr}\left(M_{2}\right) \rightarrow \cdots
$$


such that each $\operatorname{Tr}\left(M_{i}\right)$ is a finitely generated and indecomposable right $\Lambda$ module, $\hat{f}_{i} \cdots \hat{f}_{1} \hat{f}_{0} \neq 0$ for all $i$, and no $\hat{f}_{k}$ is an isomorphism. So the Proposition is proved.

According to [1, Corollary 9], if $\Lambda$ is a ring whose (projective and injective left) modules all have decompositions that complement direct summands, then $\Lambda$ is (left) artin. Thus the above results yield

Theorem. A ring $\Lambda$ is of finite representation type if (and only if) each of its left and its right modules has a decomposition that complements direct summands.

\section{REFERENCES}

1. F. W. Anderson and K. R. Fuller, Modules with decompositions that complement direct summands, J. Algebra 22 (1972), 241-253. MR 46 \#209.

2. M. Auslander, Representation dimension of Artin algebras, Queen Mary College Notes, London, 1971.

3. - Representation theory of Artin algebras. II, Communications in Algebra 1 (1974), 293-310.

4. M. Auslander and M. Bridger, Stable module theory, Mem. Amer. Math. Soc. No. 94 (1969). MR $42 \# 4580$.

5. M. Harada and Y. Sai, On categories of indecomposable modules. I, Osaka J. Math. 7 ( 1970), 323-344. MR 44 \# 4066.

6. H. Tachikawa, $Q F-3$ rings and categories of projective modules, J. Algebra 28 (1974), 408-413.

DEPARTMENT OF MATHEMATICS, UNIVERSITY OF IOWA, IOWA CITY, IOWA 52242

DEPARTMENT OF MATHEMATICS, UNIVERSITY OF TRONDHEIM, NLHT 7000, TRONDHEIM, NORWAY 